\title{
Comparison of three methods for measuring psoriasis severity in clinical studies (Part 2 of 2): use of quality of life to assess construct validity of the Lattice System Physician's Global Assessment, Psoriasis Area and Severity Index and Static Physician's Global Assessment
}

\author{
M.J. Simpson, ${ }^{1,2, \dagger}$ C. Chow, ${ }^{1, \dagger}$ H. Morgenstern, ${ }^{2,3,4}$ T.A. Luger, ${ }^{5}$ C.N. Ellis ${ }^{1, *}$ \\ ${ }^{1}$ Department of Dermatology, University of Michigan Medical School, Ann Arbor, Michigan, USA \\ ${ }^{2}$ Department of Epidemiology, School of Public Health, University of Michigan, Ann Arbor, Michigan, USA \\ ${ }^{3}$ Department of Environmental Health Sciences, School of Public Health, University of Michigan, Ann Arbor, Michigan, USA \\ ${ }^{4}$ Department of Urology, University of Michigan Medical School, Ann Arbor, Michigan, USA \\ ${ }^{5}$ Department of Dermatology, University of Münster, Münster, Germany \\ ${ }^{*}$ Correspondence: C. N. Ellis. E-mail: cellis@umich.edu
}

\begin{abstract}
Background Systems for determining psoriasis severity in clinical trials have not been sufficiently validated against patients' perceived quality of life.

Objective To validate three systems of physician-determined psoriasis severity (the Lattice System Physician's Global Assessment [LS-PGA], Psoriasis Area and Severity Index [PASI] and static Physician's Global Assessment [sPGA]).

Methods Data were from a 24-week randomized, double-blind, placebo-controlled, multicenter trial of therapy with oral calcineurin inhibitors in 445 patients. Construct validity was measured by correlations of the three severity scores with patients' self-reported quality of life (QoL) from the Dermatology Life Quality Index (DLQI) and a DLQI item about psoriasis symptoms.

Results All severity systems were moderately and positively correlated with QoL, indicating construct validity. QoL was most consistently related to physicians' assessments of body surface area involved with psoriasis (iBSA) followed by, in the order of consistency, plaque elevation, erythema and scale.

Conclusions The LS-PGA weights iBSA and aspects of plaque morphology in concert with their relative effects on QoL. The LS-PGA, SPGA and PASI are validated by their relationship to QoL in a clinical trial.

Received: 11 September 2014; Accepted: 20 October 2014

\section{Conflicts of interest}

CNE and TAL have served as consultants to Isotechnika Pharma, Inc. (now Aurinia Pharmaceuticals, Inc.), Novartis Pharmaceuticals Corp., and other manufacturers of proposed or marketed therapies for psoriasis. CNE holds a patent on the LS-PGA and has received royalties for its use. The other authors have no conflict of interest to declare.
\end{abstract}

\section{Funding sources}

Supported by the Department of Dermatology, University of Michigan Medical School. Isotechnika Pharma, Inc. sponsored the clinical trial and provided the data used in this study. TAL served as the medical director for the clinical trial; the other authors did not participate in the trial. Isotechnika Pharma, Inc. provided no support for this report nor had any role in the design, analysis, writing or submission of this work.

\section{Introduction}

A systematic review ${ }^{1}$ enumerated 53 systems for evaluating psoriasis severity in clinical trials. Because there is no agreed-upon gold standard for measuring the degree of severity of psoriasis, the validity of systems that assesses psoriasis severity cannot be

${ }^{\dagger}$ Both authors contributed equally to this work. established solely by comparing one system to another. Therefore, in this study, we validated scoring systems against patients' reported quality of life (QoL). . $^{2,3}$

Construct validation is especially important in the absence of an accepted standard. Construct validity is the extent to which a measuring system that quantifies a specific concept is associated with other measures that are expected to be related to that 
concept. If our measuring systems have construct validity in assessing 'psoriasis severity', we expect them to be associated with other measures related to severity, such as QoL of patients with psoriasis.

We used data made available to us from a phase III clinical trial to investigate the validity of the Lattice System Physician's Global Assessment (LS-PGA), Psoriasis Area and Severity Index (PASI) and static Physician's Global Assessment (sPGA) as clinical measures of psoriasis severity. The degree to which a decrease in disease severity (i.e. lower scores in each of the three clinical scoring systems) is associated with skin-related QoL improvement (i.e. lower scores in the Dermatology Life Quality Index [DLQI]) is an indication that a system has construct validity.

Content validity is the extent to which the individual components of a composite scoring system are related to the overall concept, e.g. psoriasis severity. We used the percentage of the patient's involved body surface area (iBSA) and the overall elevation, erythema and scale of the psoriasis plaques in a multiple regression analysis with DLQI to assess the relative impact of each individual component of a psoriasis severity scoring system on QoL. In addition, we wanted to find out whether the relative weighting of the components of psoriasis to obtain the final score in the LS-PGA algorithm conformed to another measure of psoriasis severity, namely QoL.

\section{Patients, materials and methods}

\section{Study design}

This observational study utilizes data from a randomized, double-blind, multicentre, phase III clinical trial (ClinicalTrials.gov, NCT00408187). For this study, we included only the 445 patients, age 18 or over, who had complete severity and QoL data at pretherapy, week 12 and week 24. Subjects were randomized in a 3:1:1 fashion to receive divided doses of voclosporin $0.8 \mathrm{mg} / \mathrm{kg} /$ day or cyclosporine $3.0 \mathrm{mg} / \mathrm{kg} /$ day ('24-week-treatment' group) or placebo ('initial-placebo' group). By protocol, all patients randomized to the placebo group crossed over to voclosporin therapy at week 12 . At pretherapy, week 12, and week 24, physicians determined sPGA and provided data to calculate $\mathrm{PASI}^{4}$ and LS-PGA ${ }^{5}$ (DATAcquire, Inc., Ann Arbor, MI, version 6.0) scores. Whenever possible, the same investigator at each site evaluated and scored the patients' severity of psoriasis throughout the trial using all three measurement systems. Patients completed the DLQI $^{6}$ at all three visits. Study investigators adhered to the Guideline for Good Clinical Practice, which is based on the Declaration of Helsinki. For additional details, see Chow et al.; our study population is the same, except for the exclusion here of ten subjects who did not have complete data for the DLQI.

\section{Lattice System Physician's Global Assessment}

Each aspect of plaque morphology (elevation, erythema, and scale) is scored on a defined 4-point ordinal scale ranging from 'none' to 'marked'; iBSA is organized into six anchored ranges. An algorithm integrates the defined ranges of iBSA with plaque morphology to produce a score on an 8-point ordinal scale ranging from 'clear' to 'very severe'. ${ }^{5}$ In determining the final LS-PGA score, iBSA defines a range of the final score, and among the three elements of plaque morphology, more weight is given to plaque elevation (the hallmark of disease activity and least influenced by external factors), some weight to erythema, and least weight to scale. ${ }^{7}$

\section{Psoriasis Area and Severity Index}

The body is divided into four portions [head (h), trunk ( $t$ ), upper (u) and lower (l) extremities], a score for surface area involved (A) and lesion characteristics [erythema (E), infiltration (I), and desquamation (D)] for each of the four areas are entered into the formula $0.1\left(E_{h}+I_{h}+D_{h}\right) A_{h}+0.2$ $\left(\mathrm{E}_{\mathrm{u}}+\mathrm{I}_{\mathrm{u}}+\mathrm{D}_{\mathrm{u}}\right) \mathrm{A}_{\mathrm{u}}+0.3\left(\mathrm{E}_{\mathrm{t}}+\mathrm{I}_{\mathrm{t}}+\mathrm{D}_{\mathrm{t}}\right) \mathrm{A}_{\mathrm{t}}+0.4\left(\mathrm{E}_{1}+\mathrm{I}_{1}+\mathrm{D}_{1}\right) \mathrm{A}_{1}$ to calculate a score from 0 to $72 .{ }^{4,7}$

\section{Static Physician's Global Assessment}

The sPGA in this study is an ordinal rating system ranging from 0 to 5 . The investigator assessed the severity of three characteristics of each patient's psoriatic plaques (induration, erythema, and scaling (IES)] averaged over all lesions. Equal weight was given to each component in determining the overall sPGA score; the investigator was instructed to assign an sPGA of 0 if IES were all 0, 1 if all IES were at least 1, 2 if all IES were at least 2, and so on. ${ }^{7}$

\section{Dermatology Life Quality Index}

The DLQI consists of ten questions dealing with the patient's perceptions of how his or her skin condition is affecting his or her QoL. ${ }^{6}$ Each item is rated on an ordinal scale of 0 to 3 leading to an overall (summed) score ranging from 0 to 30; higher scores indicate a greater adverse impact on QoL. We utilized the overall DLQI score from all ten questions and also assessed responses to Question 1, which is the only question directly about skin symptoms of psoriasis, inquiring about how itchy, sore, painful or stinging the patient's skin has been.

The DLQI has been used extensively in psoriasis research. Construct validity of the DLQI has been established with generic, dermatologic-specific and disease-specific measures in over 35 studies. Furthermore, test-retest reliability and internal consistency have been shown to be high in multiple studies. ${ }^{8}$

\section{Statistical analysis}

For analysis, we assigned numerical scores for LS-PGA and sPGA as in Chow et al. ${ }^{7}$ To analyse the associations between the three clinical measures and the patient-reported skin-related QoL, we calculated Kendall-Stuart's tau- $\mathrm{c}^{9}$ at each time point between each of the clinical severity scores and the total DLQI score and the score for DLQI Question 1 for symptoms. Kendall-Stuart's 
tau-c is a useful measure of correlation for ordinal variables with appreciable ties and large contingency tables. Additionally, we calculated tau-c between the change in each severity score and the change in the DLQI score from pretherapy to week 24.

We used multiple linear regression analysis to investigate the relation between the iBSA or plaque characteristics (erythema, elevation, and scale) and the DLQI score. We used components of the LS-PGA because they are scored by the physician for the patient's entire body. Because erythema, elevation and scale were scored on a 4-point ordinal scale in the LS-PGA, we also categorized iBSA as a 4-point ordinal scale by condensing the LS-PGA ranges of iBSA into the following groups: $0 \%, 1 \%$ to $9 \%, 10 \%$ to $29 \%$ and $>29 \%$. A model was fitted to patients in the 24 -weektreatment or initial-placebo groups at each of the three time points (6 models) with the four aspects of psoriasis as predictors. Analyses used SAS version 9.2.1 (SAS Institute, Cary, NC, USA).

\section{Results}

Of the 445 subjects with complete data, 360 were randomized to the 24-week-treatment group and 85 to the initial-placebo group; the groups were similar (Table 1). All three psoriasis severity scores were positively correlated (Table 2) with the total DLQI score and with the score for certain skin symptoms (Item 1 of the DLQI that inquires about how itchy, sore, painful or stinging the patient's skin has been in the prior 7 days). The correlations among the QoL measures and the severity scores were weak at pretherapy (tau-c $=0.08-0.32$ ) but higher at week 12 and week 24 (tau-c $=0.25-0.54$ ). The change in each severity score was positively correlated with the change in DLQI score from pretherapy to week 24 (tau-c $=0.22-0.34$, Table 3 ).

Table 4 presents the coefficients from the multiple linear regression models that estimate the impact of scale, erythema, elevation and iBSA as predictors on the DLQI score at each observation. Each value represents the estimated mean difference in the DLQI score for a one-category increase in the LS-PGA score of iBSA or plaque scale, erythema or elevation. The most consistent positive predictor of DLQI score was iBSA. During active therapy, elevation had a greater impact than erythema. Throughout the study, scale was only weakly and often inversely associated with the QoL score.

\section{Discussion}

\section{Construct validity}

Because they address common and important signs of psoriasis, all three measures appear to measure psoriasis severity (i.e. they have face validity). Using data from a phase III clinical trial, each of the physician-scoring systems also demonstrated construct validity in determining psoriasis severity. That is, the measures of psoriasis severity are positively correlated with measures of QoL prior to and especially during and at the conclusion of a clinical trial (Table 2). We would not expect perfect correlation
Table 1 Distribution of clinical psoriasis severity and QoL scores at pretherapy, by assigned treatment group. There were no significant differences in pretherapy characteristics between the 24-week-treatment group (patients who received active therapy throughout the study) and initial-placebo group (patients who received placebo for 12 weeks and active therapy for 12 weeks). Psoriasis Area and Severity Index has a range of 0-72. Lower values of DLQI indicate better QoL. Based on others' suggestions, ${ }^{10,11}$ we divided PASI and DLQI into the segments shown.

\begin{tabular}{|c|c|c|c|}
\hline & Total $(n=445)$ & $\begin{array}{l}\text { 24-Week- } \\
\text { Treatment } \\
(n=360)\end{array}$ & $\begin{array}{l}\text { Initial-Placebo } \\
(n=85)\end{array}$ \\
\hline \multicolumn{4}{|c|}{ Psoriasis Area and Severity Index, mean (SD) } \\
\hline & $18.5(7.0)$ & $18.6(7.1)$ & $18.0(6.4)$ \\
\hline \multicolumn{4}{|c|}{ Ranges of Psoriasis Area and Severity Index, $n(\%)$} \\
\hline$<7$ & $0(0)$ & $0(0)$ & $0(0.0)$ \\
\hline $7-12$ & $71(16)$ & $57(16)$ & $14(16)$ \\
\hline$>12$ & $374(84)$ & $303(84)$ & $71(84)$ \\
\hline \multicolumn{4}{|c|}{ Static Physician's Global Assessment, $n(\%)$} \\
\hline 0 & $0(0)$ & $0(0)$ & $0(0)$ \\
\hline 1 & $0(0)$ & $0(0)$ & $0(0)$ \\
\hline 2 & $3(1)$ & $3(1)$ & $0(0.0)$ \\
\hline 3 & $254(57)$ & $202(56)$ & $52(61)$ \\
\hline 4 & $170(38)$ & $137(38)$ & $33(39)$ \\
\hline 5 & $18(4)$ & $18(5)$ & $0(0)$ \\
\hline \multicolumn{4}{|c|}{ Lattice System Physician's Global Assessment, $n$ (\%) } \\
\hline Clear & $0(0)$ & $0(0)$ & $0(0)$ \\
\hline Almost-clear & $0(0)$ & $0(0)$ & $0(0)$ \\
\hline Mild & $0(0)$ & $0(0)$ & $0(0)$ \\
\hline Mild to Moderate & $0(0)$ & $0(0)$ & $0(0)$ \\
\hline Moderate & $13(3)$ & $10(2)$ & $3(4)$ \\
\hline Moderate to Severe & $122(27)$ & $98(27)$ & $24(28)$ \\
\hline Severe & $193(43)$ & $159(44)$ & $34(40)$ \\
\hline Very Severe & $117(26)$ & $93(26)$ & $24(28)$ \\
\hline \multicolumn{4}{|c|}{ Dermatology Life Quality Index, mean (SD) } \\
\hline & $11(6)$ & $11(6)$ & $11(7)$ \\
\hline \multicolumn{4}{|c|}{ Ranges of Dermatology Life Quality Index, $n(\%)$} \\
\hline $0-1$ & $164(37)$ & $159(44)$ & $5(6)$ \\
\hline $2-5$ & $128(29)$ & $103(29)$ & $25(29)$ \\
\hline $6-10$ & $86(19)$ & $66(18)$ & $20(24)$ \\
\hline $11-20$ & 59 (13) & $30(8)$ & 29 (34) \\
\hline $21-30$ & $8(2)$ & $2(1)$ & $6(7)$ \\
\hline
\end{tabular}

$\mathrm{SD}$, standard deviation.

(tau-c $=1$ ) between physician-determined severity scores and the patient's self-rated QoL because they reflect different underlying constructs. However, our moderate positive correlations indicate that when psoriasis severity is lower as measured by the physician rating systems, this occurs in concert with better skinrelated patient QoL. Additionally, the change in psoriasis severity during the trial was positively correlated with the change in QoL score (Table 3), again indicating that as psoriasis severity decreases by physician measures, it is associated with QoL improvement among patients. 
Table 2 Correlation between the Lattice System Physician's Global Assessment (LS-PGA), Psoriasis Area and Severity Index (PASI) and static Physician's Global Assessment (sPGA) scores and the Dermatology Life Quality Index (DLQI) score and the DLQI symptom severity question at pretherapy, week 12, week 24 and by the assigned treatment group. Question 1 of the DLQI inquires about how itchy, sore, painful or stinging the patient's skin has been in the prior seven days.

\begin{tabular}{|c|c|c|c|c|c|c|}
\hline & \multicolumn{2}{|l|}{ LS-PGA } & \multicolumn{2}{|l|}{ PASI } & \multicolumn{2}{|l|}{ sPGA } \\
\hline & $\begin{array}{l}\text { 24-Week- } \\
\text { Treatment } \\
(n=360)\end{array}$ & $\begin{array}{l}\text { Initial-Placebo* } \\
(n=85)\end{array}$ & $\begin{array}{l}\text { 24-Week- } \\
\text { Treatment } \\
(n=360)\end{array}$ & $\begin{array}{l}\text { Initial-Placebo* } \\
(n=85)\end{array}$ & $\begin{array}{l}\text { 24-Week- } \\
\text { Treatment } \\
(n=360)\end{array}$ & $\begin{array}{l}\text { Initial-Placebo* } \\
(n=85)\end{array}$ \\
\hline \multicolumn{7}{|l|}{ Pretherapy } \\
\hline DLQI & $0.10 \dagger$ & $0.32 \dagger$ & $0.14 \dagger$ & 0.15 & $0.13 \dagger$ & $0.26 \dagger$ \\
\hline Question 1 & 0.08 & 0.13 & $0.13 \dagger$ & 0.10 & $0.12 \dagger$ & 0.17 \\
\hline \multicolumn{7}{|l|}{ Week 12} \\
\hline DLQI & $0.43 \dagger$ & $0.35 \dagger$ & $0.40 \dagger$ & $0.40 \dagger$ & $0.39 \dagger$ & $0.41 \dagger$ \\
\hline Question 1 & $0.44 \dagger$ & $0.42 \dagger$ & $0.45 \dagger$ & $0.54 \dagger$ & $0.39 \dagger$ & $0.43 \dagger$ \\
\hline \multicolumn{7}{|l|}{ Week 24} \\
\hline DLQI & $0.41 \dagger$ & $0.41 \dagger$ & $0.38 \dagger$ & $0.36 \dagger$ & $0.37 \dagger$ & $0.29 \dagger$ \\
\hline Question 1 & $0.38 \dagger$ & $0.44 \dagger$ & $0.42 \dagger$ & $0.44 \dagger$ & $0.37 \dagger$ & $0.25 \dagger$ \\
\hline
\end{tabular}

*Initial-Placebo group crossed over to active therapy at week 12.

$\dagger P<0.05$ from testing the null hypothesis that there is no association (tau-c $=0$ ).

Table 3 Correlation between change in the Lattice System Physician's Global Assessment, Psoriasis Area and Severity Index, and static Physician's Global Assessment scores and the change in the Dermatology Life Quality Index score from pretherapy to week 24 , by assigned treatment group $(n=445)$

\begin{tabular}{lll} 
& $\begin{array}{l}\text { 24-Week-Treatment } \\
(\boldsymbol{n}=\mathbf{3 6 0})\end{array}$ & $\begin{array}{l}\text { Initial-Placebo* } \\
(\boldsymbol{n}=\mathbf{8 5})\end{array}$ \\
$\begin{array}{lll}\text { Lattice System Physician's } \\
\text { Global Assessment }\end{array}$ & 0.23 & 0.32 \\
\hline $\begin{array}{l}\text { Psoriasis Area and } \\
\text { Severity Index }\end{array}$ & 0.22 & 0.24 \\
\hline $\begin{array}{l}\text { Static Physician's Global } \\
\text { Assessment }\end{array}$ & 0.23 & 0.34 \\
\hline
\end{tabular}

*Initial-Placebo group crossed over to active therapy at week 12. $P<0.05$ for all values.

The correlations for all three clinical severity scores and QoL are considerably stronger at weeks 12 and 24 than at pretherapy (Table 2). This pattern, similar to that reported by Shikiar et al., ${ }^{12}$ is probably due to reduced variability in psoriasis severity at pretherapy because of the effects of enrolment criteria that narrow the range of patient severity at the initial visit. Because correlation coefficients are variance-based measures of association, the smaller variance in psoriasis severity relative to DLQI variance at pretherapy results in a lower correlation compared to later visits, even when the linear relation (slope) remains constant. Similarly, the correlation between Item 1 of the DLQI and each overall severity score is stronger at weeks 12 and 24 than at pretherapy.

Psoriasis of equal severity (as rated by physicians) conforms to a range of patient-reported QoL scores and symptoms. Clinicians are familiar with patients who have large amounts of psoriasis yet are unfazed; conversely, some patients are deeply troubled by relatively few areas of involvement. These examples support the role of investigating measures of both QoL and clinical psoriasis severity in research studies; they capture distinct information. ${ }^{13,14}$ Nevertheless, in large groups of patients with psoriasis in clinical trials, QoL is closely related to clinical severity. ${ }^{15}$ We are unaware of any clinical trial in which clinical severity scores improved overall without concomitant improvement in overall QoL.

\section{Content validity}

Using patients' self-reports of skin-related QoL, we investigated the content validity of the physician-measured extent of psoriasis involvement and plaque morphology. We found that iBSA was the attribute of psoriasis that most consistently associated with DLQI scores. Thus, it may surprise that the sPGA does not incorporate any assessment of iBSA. In contrast, iBSA is the primary component of the LS-PGA algorithm and an important aspect of the PASI calculation. ${ }^{4,5}$

The amount of scale on the psoriasis plaques as determined by the physician was minimally and inconsistently associated with DLQI scores. Although patients may mention scale as part of the disease process that adversely affects their life, the physician rating of scale is not a good predictor of the patient's QoL (Table 4). This may be in part because scale varies with factors such as skin hydration, application of topical moisturizers or ambient conditions. Furthermore, the amount of scale as a static measure at a patient visit may not reflect scaling (the flaking off) that may be even more distressing to patients.

In contrast to scale, elevation and erythema were positively associated with the DLQI score at all observations, although the associations were not consistently strong, particularly at pretherapy (Table 4). With active therapy, change in plaque elevation had more effect on QoL than did erythema. When translating these 
Table 4 Estimated mean difference in Dermatology Life Quality Index (DLQI) score for one category increase in the Lattice System Physician's Global Assessment (LS-PGA) score. The values for erythema, elevation, scale, and involved body surface area were obtained at pretherapy, week 12 , and week 24 , and are shown by the assigned treatment group.

\begin{tabular}{|c|c|c|c|c|c|c|}
\hline & \multicolumn{3}{|l|}{ Treatment $(n=360)$} & \multicolumn{3}{|c|}{ Initial-Placebo* $(n=85)$} \\
\hline & $\begin{array}{l}\text { Estimated mean } \\
\text { difference in DLQI }\end{array}$ & $\begin{array}{l}95 \% \text { confidence } \\
\text { interval }\end{array}$ & $P \dagger$ & $\begin{array}{l}\text { Estimated mean } \\
\text { difference in DLQI }\end{array}$ & $\begin{array}{l}95 \% \text { confidence } \\
\text { interval }\end{array}$ & $P_{\dagger}$ \\
\hline \multicolumn{7}{|l|}{ Pretherapy } \\
\hline Scale & -0.4 & $(-1.8,0.9)$ & 0.51 & 0.2 & $(-2.6,3.1)$ & 0.87 \\
\hline Erythema & 1.1 & $(-0.2,2.4)$ & 0.10 & 2.1 & $(-0.7,4.9)$ & 0.13 \\
\hline Elevation & 0.6 & $(-0.8,1.9)$ & 0.42 & 1.0 & $(-1.6,3.5)$ & 0.45 \\
\hline Body Surface Area & 1.7 & $(0.3,3.1)$ & 0.02 & 4.0 & $(0.9,7.1)$ & 0.01 \\
\hline \multicolumn{7}{|l|}{ Week 12} \\
\hline Scale & -0.6 & $(-1.5,0.4)$ & 0.22 & 1.0 & $(-1.8,3.7)$ & 0.48 \\
\hline Erythema & 0.4 & $(-0.5,1.3)$ & 0.34 & 1.3 & $(-1.5,4.2)$ & 0.36 \\
\hline Elevation & 2.2 & $(1.3,3.2)$ & $<0.01$ & 0.2 & $(-2.6,3.1)$ & 0.87 \\
\hline Body Surface Area & 1.2 & $(0.6,1.8)$ & $<0.01$ & 3.0 & $(0.9,5.0)$ & $<0.01$ \\
\hline \multicolumn{7}{|l|}{ Week 24} \\
\hline Scale & -0.1 & $(-1.1,0.9)$ & 0.83 & -3.2 & $(-5.6,-0.8)$ & 0.01 \\
\hline Erythema & 0.5 & $(-0.4,1.5)$ & 0.27 & 2.6 & $(0.2,5.0)$ & 0.03 \\
\hline Elevation & 1.0 & $(0.0,1.9)$ & 0.05 & 3.1 & $(0.5,5.6)$ & 0.02 \\
\hline Body Surface Area & 1.6 & $(1.0,2.3)$ & $<0.01$ & 1.9 & $(0.6,3.2)$ & $<0.01$ \\
\hline
\end{tabular}

*Initial-placebo group crossed over to active therapy at week 12.

$\uparrow P$ value testing the null hypothesis of no association between each characteristic and the DLQI score, adjusting for the other characteristics.

findings to the validation of scoring systems, a scoring system that weights elevation, erythema, and scale equally would not capture the varying degrees to which these factors affect the patient's rating of QoL. PASI and sPGA have equal weighting for all characteristics; the LS-PGA weights elevation, erythema and scale ${ }^{5}$ in concert with their relative associations with QoL.

Our study benefited from the large sample size and longitudinal design. Subjects were examined three times during the study - when all were not under treatment, when some had been on active treatment for 12 weeks and when all subjects had been on active treatment for at least 12 weeks. Thus, we have data from subjects with varying psoriasis severity at various times on treatment.

Hampering any study of the validity of measures of psoriasis severity is the lack of a 'gold-standard' for determining the severity of psoriasis and response to treatment. While this is an inherent limitation with no known remedy, it means we must rely on assessments of construct validity to evaluate and compare severity measures. We used the DLQI, the most widely used measure of QoL in dermatology studies, as a validated measure of skinrelated QoL to establish the construct validity of physiciandetermined measures of severity. ${ }^{16,17}$ In this clinical trial, the DLQI was the only evaluation of QoL; thus, our analysis was limited to the DLQI, and we chose Question 1 from the DLQI because of its relevance to psoriasis severity measurement systems. Other measures of QoL exist, and some are specific to psoriasis; we hope that large clinical trials that utilize other measures of QoL may become available to us or other researchers in future. Because more than $95 \%$ of the patients in the trial were white, our results may not generalize to all patients.

When choosing a severity measure for a clinical trial of psoriasis patients, a measure that is known to be reliable, valid and responsive to changes in severity is desired. Because patients put trust in their dermatologists' treatment recommendations with the hope of years of improved QoL, it is important to have valid measures so that physicians may choose therapeutic approaches based on interpretable clinical trial results. Although there are various reasons for choosing among rating systems of psoriasis severity in clinical trials, ${ }^{7}$ our results indicate that the measurement of extent of psoriasis (i.e. iBSA) should be an important component of the evaluation method because of its relevance to patients' QoL. Similarly, appropriate weighting of psoriasis plaque components (e.g. elevation, erythema and scale), such as that which occurs in the LS-PGA, may aid in determining a clinical severity score that closely corresponds to the quality of life of patients with psoriasis.

\section{Acknowledgments}

We thank Isotechnika Pharma, Inc. for providing the data used in this report and we are grateful to the investigators who performed the clinical trial (for a list, see Ref. 7) and the patients who participated in this study.

\section{References}

1 Spuls PI, Lecluse LL, Poulsen ML, Bos JD, Stern RS, Nijsten T. How good are clinical severity and outcome measures for psoriasis?: quantitative evaluation in a systematic review. J Invest Dermatol 2010; 130: 933-943. 
2 Rapp SR, Feldman SR, Exum ML, Fleischer AB Jr, Reboussin DM. Psoriasis causes as much disability as other major medical diseases. J Am Acad Dermatol 1999; 41: 401-407.

3 Koo J. Population-based epidemiologic study of psoriasis with emphasis on quality of life assessment. Dermatol Clin 1996; 14: 485-496.

4 Fredriksson T, Pettersson U. Severe psoriasis_oral therapy with a new retinoid. Dermatologica 1978; 157: 238-244.

5 Langley RG, Ellis CN. Evaluating psoriasis with Psoriasis Area and Severity Index, Psoriasis Global Assessment, and Lattice System Physician's Global Assessment. J Am Acad Dermatol 2004; 51: 563-569.

6 Finlay AY, Khan GK. Dermatology Life Quality Index (DLQI) - a simple practical measure for routine clinical use. Clin Exp Dermatol 1994; 19: 210-216.

7 Chow C, Simpson MJ, Luger TA, Chubb H, Ellis CN. Comparison of three methods for measuring psoriasis severity in clinical studies (Part 1 of 2): change during therapy in Psoriasis Area and Severity Index, Static Physician's Global Assessment, and Lattice System Physician’s Global Assessment. JEADV 2015; in press.

8 Basra MKA, Fenech R, Gatt RM, Salek MS, Finlay AY. The dermatology life quality index 1994-2007: a comprehensive review of validation data and clinical results. Br J Dermatol 2008; 159: 997-1035.

9 Stuart A. The estimation and comparison of strengths of association in contingency tables. Biometrika 1953; 40: 105-110.

10 Schmitt J, Wozel G. The Psoriasis Area and Severity Index is the adequate criterion to define severity in chronic plaque-type psoriasis. Dermatol 2005; 210: 194-199.
11 Hongbo Y, Thomas CL, Harrison MA, Salek MS, Finlay AY. Translating the science of quality of life into practice: what do dermatology life quality index scores mean? J Invest Dermatol 2005; 125: 659-664.

12 Shikiar R, Willian MK, Okun MM, Thompson CS, Revicki DA. The validity and responsiveness of three quality of life measures in the assessment of psoriasis patients: results of a phase II study. Health Qual Life Outcomes 2006; 4: 71.

13 Sampogna F, Sera F, Abeni D and the IDI Multipurpose Psoriasis Research on Vital Experiences (IMPROVE) Investigators. Measures of clinical severity, quality of life, and psychological distress in patients with psoriasis: a cluster analysis. J Invest Dermatol 2004; 122: 602-607.

14 Chularojanamontri L, Griffiths CEM, Chalmers RJG. The Simplified Psoriasis Index (SPI): a practical tool for assessing psoriasis. J Invest Dermatol 2013; 133: 1956-1962.

15 Mattei PL, Corey KC, Kimball AB. Psoriasis Area Severity (PASI) and the Dermatology Life Quality Index (DLQI): the correlation between disease severity and psychological burden in patients treated with biological therapies. J Eur Acad Dermatol Venereol 2014; 28: 333-337.

16 Bronsard V, Paul C, Prey S et al. What are the best outcome measures for assessing quality of life in plaque type psoriasis? A systematic review of the literature. J Eur Acad Dermatol Venereol 2010; 24(Suppl 2): 17-22.

17 Mazzotti E, Picardi A, Sampogna F, Sera F, Pasquini P, Abeni D and the IDI Multipurpose Psoriasis Research on Vital Experiences (IMPROVE) Study Group. Sensitivity of the Dermatology Life Quality Index to clinical change in patients with psoriasis. Br J Dermatol. 2003; 149: 318-322. 